\title{
Correlation between optic nerve head circulation and visual function before and after anti-VEGF therapy for central retinal vein occlusion: prospective, interventional case series
}

Daisuke Nagasato ${ }^{1,2}$, Yoshinori Mitamura $^{3^{*}}$, Kentaro Semba ${ }^{3}$, Kei Akaiwa ${ }^{3}$, Toshihiko Nagasawa ${ }^{1,2}$, Yuki Yoshizumi $^{1}$, Hitoshi Tabuchi ${ }^{1}$ and Yoshiaki Kiuchi ${ }^{2}$

\begin{abstract}
Background: To determine the correlation between the optic nerve head $(\mathrm{ONH})$ circulation determined by laser speckle flowgraphy and the best-corrected visual acuity or retinal sensitivity before and after intravitreal bevacizumab or ranibizumab for central retinal vein occlusion.
\end{abstract}

Methods: Thirty-one eyes of 31 patients were treated with intravitreal bevacizumab or ranibizumab for macular edema due to a central retinal vein occlusion. The blood flow in the large vessels on the $\mathrm{ONH}$, the best-corrected visual acuity, and retinal sensitivity were measured at the baseline, and at 1,3, and 6 months after treatment. The arteriovenous passage time on fluorescein angiography was determined. The venous tortuosity index was calculated on color fundus photograph by dividing the length of the tortuous retinal vein by the chord length of the same segment. The blood flow was represented by the mean blur rate (MBR) determined by laser speckle flowgraphy. To exclude the influence of systemic circulation and blood flow in the ONH tissue, the corrected MBR was calculated as MBR of ONH vessel area - MBR of ONH tissue area in the affected eye divided by the vascular MBR - tissue MBR in the unaffected eye. Pearson's correlation tests were used to determine the significance of correlations between the MBR and the best-corrected visual acuity, retinal sensitivity, arteriovenous passage time, or venous tortuosity index.

Results: At the baseline, the corrected MBR was significantly correlated with the arteriovenous passage time and venous tortuosity index ( $r=-0.807, P<0.001 ; r=-0.716, P<0.001$; respectively). The corrected MBR was significantly correlated with the best-corrected visual acuity and retinal sensitivity at the baseline, and at 1, 3, and 6 months (all $P<0.050$ ). The corrected MBR at the baseline was significantly correlated with the best-corrected visual acuity at 6 months $(r=-0.651$, $P<0.001)$ and retinal sensitivity at 6 months $(r=0.485, P=0.005)$.

Conclusions: The pre-treatment blood flow velocity of ONH can be used as a predictive factor for the best-corrected visual acuity and retinal sensitivity after anti-VEGF therapy for central retinal vein occlusion.

Trial registration: Trial Registration number: UMIN000009072. Date of registration: 10/15/2012.

Keywords: Anti-vascular endothelial growth factor agent, Central retinal vein occlusion, Fundus-related microperimetry, Laser speckle flowgraphy, Retinal blood flow

\footnotetext{
* Correspondence: ymitaymitaymita@yahoo.co.jp

${ }^{3}$ Department of Ophthalmology, Institute of Biomedical Sciences, Tokushima

University Graduate School, 3-18-15 Kuramoto, Tokushima 770-8503, Japan

Full list of author information is available at the end of the article
} 


\section{Background}

A central retinal vein occlusion (CRVO) is one of the major causes of vision reduction, and anti-vascular endothelial growth factor (VEGF) agents have been shown to significantly improve visual acuity in eyes with macular edema (ME) due to a CRVO [1].

Laser speckle flowgraphy (LSFG) is a non-invasive method of real-time measurements of the blood flow on the optic nerve head $(\mathrm{ONH})$, retina, and choroid [2-6]. It can measure the relative blood flow velocity, called the mean blur rate (MBR), that has been shown to be significantly correlated with the actual blood flow rate determined by the hydrogen gas clearance method and the microspheres technique $[7,8]$. It has been reported that LSFG can record the blood flow from the same location of an eye with high reproducibility especially on the $\mathrm{ONH}$ [9]. This then provides an accurate way to monitor the circulation changes before and after pharmacological interventions [10].

Yamada et al. reported that the MBR values in large $\mathrm{ONH}$ vessels measured by LSFG were correlated with the higher aqueous VEGF concentrations in eyes with CRVO [11]. Nagaoka et al. reported that a single intravitreal bevacizumab (IVB) injection did not affected the retinal microcirculation in eyes with acute branch retinal vein occlusion (BRVO) for at least 3 months after the injection [12]. However, there has been no report that compared the ocular circulation before and after antiVEGF therapy for CRVO except for a report of 3 cases in which the statistical association between $\mathrm{ONH}$ circulation and visual function was not determined [13].

Thus, the purpose of this study was to examine the relationship between the retinal blood flow and visual acuity or retinal sensitivity in 31 eyes before and after IVB or intravitreal ranibizumab (IVR) for CRVO.

\section{Methods}

This was a prospective, interventional case series of 31 eyes of 31 treatment-naïve patients $(20$ men and 11 women) with unilateral CRVO. The patients were examined by LSFG and microperimetry at the baseline and at 1,3 , and 6 months after IVB or IVR for ME due to a CRVO. The fluorescein arteriovenous passage time was determined at the baseline. All patients were examined within 3 month of the onset of the symptoms. The age of the patients at presentation ranged from 40 to 83 years (mean, 66.9 years). All treatment-naïve patients who were diagnosed with ME due to CRVO, had a healthy fellow eye, and had IVB or IVR injections in the Department of Ophthalmology of Saneikai Tsukazaki Hospital from October 2012 through February 2015 were studied. Approval was obtained from the Institutional Review Board of Saneikai Tsukazaki Hospital prior to beginning this study, and the patients gave their written informed consent prior to their inclusion. The patients have provided permission to publish clinical data of their case in this study. This study was registered with the University hospital Medical Information Network (UMIN) clinical trials registry. The registration title is "UMIN000009072, Correlation between optic nerve head circulation and retinal sensitivity before and after antiVEGF drug intravitreal injection for central retinal vein occlusion" (October 15, 2012). The procedures used in this study adhered to the tenets of the Declaration of Helsinki.

Patients with ME due to a CRVO, central foveal thickness of $\geq 250 \mu \mathrm{m}$ in the optical coherence tomographic (OCT) images, and a decimal visual acuity from 0.01 to 0.8 were studied. Patients with a history of cerebral infarction, anti-VEGF therapy, vitrectomy, and uveitis, or other vitreoretinal diseases were excluded. In addition, patients with uncontrolled high blood pressure, diabetes, intraocular pressure (IOP) of $21 \mathrm{mmHg}$ or more, or iris neovascularization were excluded.

The blood flow of the major blood vessels on the $\mathrm{ONH}$ was measured in all patients, and the arteriovenous passage time of the retina was determined by fluorescein angiography (FA). A previous study investigating CRVO with FA [14] showed that more than 10 disc areas of the nonperfusion areas were observed in the ischemic type of CRVO. Thus, a diagnosis of ischemic CRVO was made in eyes with more than 10 disc areas of the nonperfusion areas [11]. The venous tortuosity index was calculated on color fundus photograph.

Patients who started their treatment between October 2012 and August 2013 received IVB injections $(1.25 \mathrm{mg} /$ $0.05 \mathrm{~mL}$ ), and those who started treatment between September 2013 and February 2015 received IVR injections $(0.5 \mathrm{mg} / 0.05 \mathrm{~mL})$. After the initial treatment, the patients were examined once a month. When the central foveal thickness was $\geq 250 \mu \mathrm{m}$, or the physician determined that additional treatment was necessary, the patients received additional injections of the same antiVEGF agent.

All patients had a standard ophthalmologic examination including IOP measurements before and after IVB or IVR. The best-corrected visual acuity (BCVA) was measured with a standard Japanese Landolt visual acuity chart, and the decimal visual acuity was converted to the logarithm of the minimal angle of resolution (logMAR) units for statistical analyses. The anterior and posterior segments were examined by slit-lamp biomicroscopy, indirect ophthalmoscopy, color fundus photography, and spectral-domain OCT (SD-OCT). The systolic blood pressure (SBP), diastolic blood pressure (DBP), and heart rate were measured. The mean arterial pressure (MAP) and the mean ocular perfusion pressure (MOPP) were calculated according to the following formulas and used for the analyses: $\mathrm{MAP}=$ $\mathrm{DBP}+1 / 3(\mathrm{SBP}-\mathrm{DBP})$, and MOPP = 2/3 MAP $-\mathrm{IOP}$. 


\section{Laser speckle flowgraphy}

LSFG-NAVI (Softcare, Fukuoka, Japan) was used to evaluate the $\mathrm{ONH}$ circulation in eyes with a CRVO (Figs. 1 and 2). The principles of LSFG have been reported in detail [15]. In brief, LSFG uses a diode laser (wavelength $830 \mathrm{~nm}$ ) to detect the movement of the red blood cells in the blood vessels. The light scattered by the movement of the blood cells creates speckle patterns on the area where the sensor is focused, and the scattered light produces a blur in the speckle patterns. The mean blur rate, which is the change in the blur, is a quantitative value that represents the relative blood flow velocity [15-19].

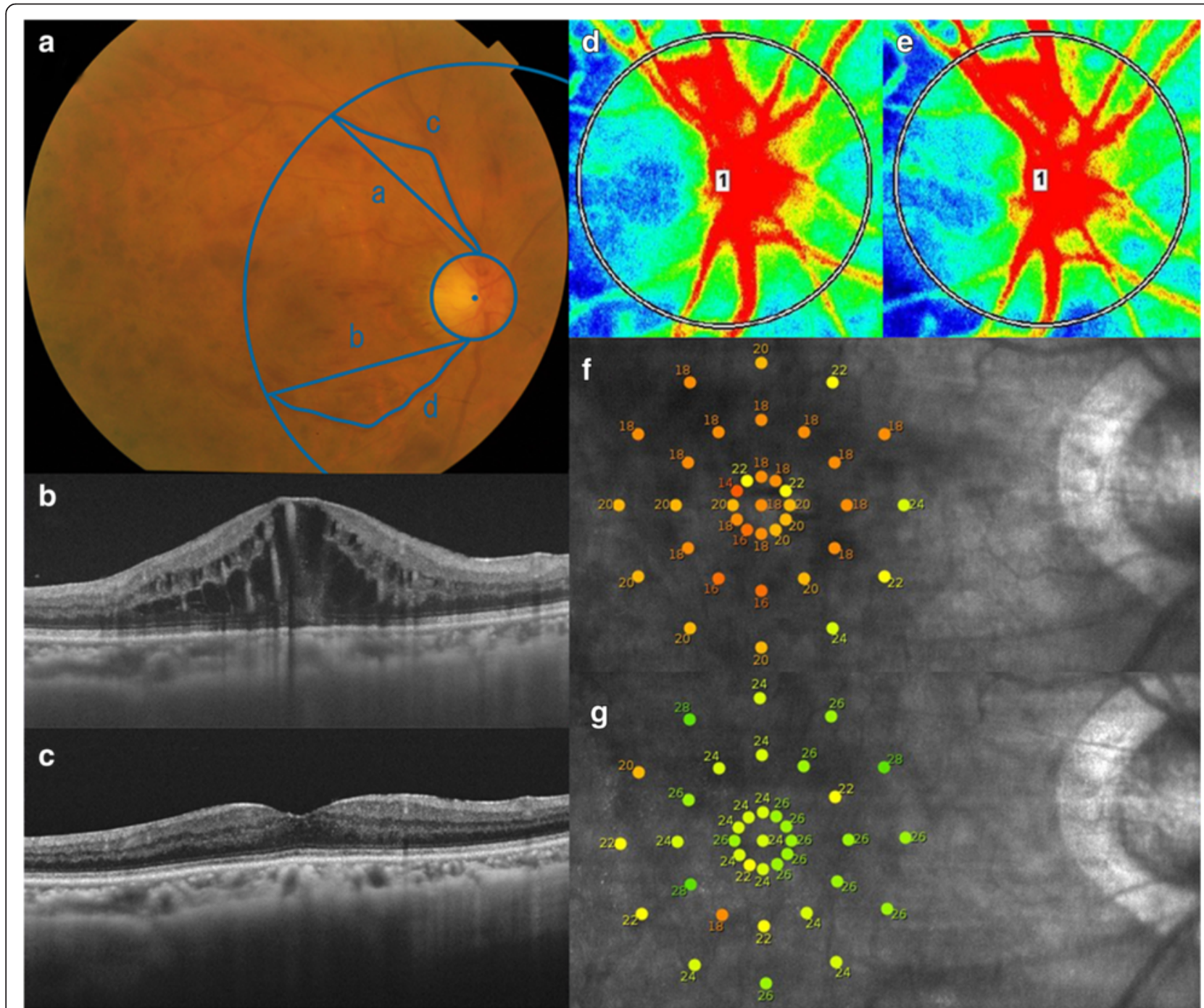

Fig. 1 Ophthalmologic examination images before and 6 months after anti-VEGF therapy for central retinal vein occlusion. Fundus photograph, spectral-domain optical coherence tomographic (SD-OCT) images, laser speckle flowgraphic (LSFG) images, and microperimetric maps before and 6 months after an initial anti-VEGF therapy of the right eye of a 66-year-old woman with cystoid macular edema (CME) due to a central retinal vein occlusion are presented. The decimal best-corrected visual acuity was 0.5 before the treatment and 1.0 at 6 months after the treatment. a: Fundus photograph at the baseline. The venous tortuosity index was calculated on color fundus photograph. Measurements of superior and inferior venous arcades were obtained starting from the optic disc margin to the crossing point of a circle whose diameter is the distance from the center of optic disc to the fovea. The course of the veins was traced using Photoshop (Adobe Systems, Inc. Ca, USA). NIH ImageJ software was used to measure the lengths of the tortuous vein ( $c$ and d) and chord of the vessels ( $a$ and b). The venous tortuosity was calculated by dividing the length of the tortuous retinal vein by the chord length of the same segment (c/a and d/b). The average of the venous tortuosity $((c / a+d / b) / 2)$ was calculated to obtain the venous tortuosity index. b: SD-OCT image at the baseline showing CME. c: SD-OCT image at 6 months showing a resolution of the CME. $\mathbf{d}$ : A false-color composite map of the optic nerve head was created using the LSFG findings at the baseline. The red area indicates a faster blood flow, and the blue area indicates a slower blood flow. e: A false-color composite map by LSFG at 6 months. There is no obvious difference in the blood flow as compared with the LSFG map at the baseline (d). f: Microperimetric map image at the baseline. A total of 37 stimulus locations covering the central $10^{\circ}$ field were tested. The mean retinal sensitivity at the 37 locations is $19.1 \mathrm{~dB}$. $\mathbf{~ g : ~ M i c r o p e r i m e t r i c ~}$ map image at 6 months. The mean retinal sensitivity at the 37 locations is $24.5 \mathrm{~dB}$ 


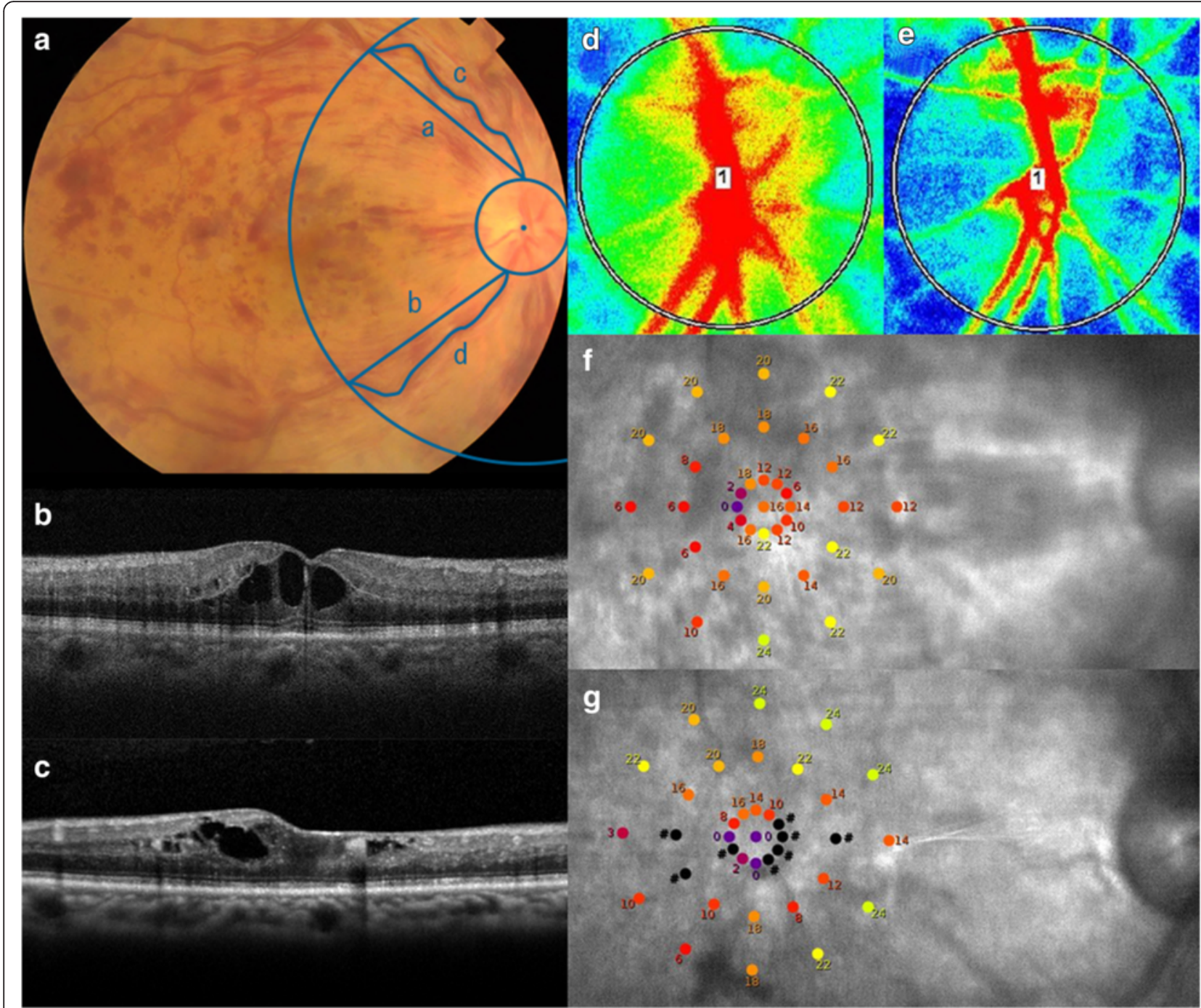

Fig. 2 Ophthalmologic examination images before and 6 months after anti-VEGF therapy for central retinal vein occlusion. Fundus photograph, spectral-domain optical coherence tomographic (SD-OCT) images, laser speckle flowgraphic (LSFG) images, and microperimetric maps before and 6 months after an initial anti-VEGF therapy of the right eye of a 66-year-old man with cystoid macular edema (CME) due to a central retinal vein occlusion are presented. The decimal best-corrected visual acuity was 0.3 before the treatment and 0.1 at 6 months after the treatment. a: Fundus photograph at the baseline. Note that the venous tortuosity is larger as compared with fundus photograph presented in Fig. 1a. b: SD-OCT image at the baseline shows CME. c: SD-OCT image at 6 months indicates residual CME and retinal thinning around the fovea. d: A false-color composite map at the optic nerve head was created using LSFG at the baseline. The red area indicates a faster blood flow, and the blue area indicates a slower blood flow. e: A false-color composite map by LSFG at 6 months. Note the decrease of blood flow as compared with the LSFG map before the treatment (d). f: Microperimetric map at the baseline. The mean retinal sensitivity at the 37 locations is $14.4 \mathrm{~dB}$. g: Microperimetric map image at 6 months. The mean retinal sensitivity at the 37 locations is $10.6 \mathrm{~dB}$

The patient's pupil was dilated with Mydrin P (1\% tropicamide and $2.5 \%$ phenylephrine) $30 \mathrm{~min}$ prior to the LSFG examinations. During the examinations, the patients were instructed to fixate steadily on a target light while the speckle pattern on the $\mathrm{ONH}$ was recorded. With an auto-tracking system, the same site can be measured for several seconds. The relative blood flow velocity is represented by the MBR and displayed as a two-dimensional color map. The MBR of the vessel area is expressed as the MV and the MBR of the tissue area as the MT. The appropriate threshold between the vessel and tissue areas is automatically determined by the built-in software, and the MBR of the area of the major arteries and veins as MV and the MBR of the tissue area as MT can be calculated (Fig. 3) [9].

When measuring the retinal circulation with LSFG, the measurements are influenced by the deep choroidal circulation. Similarly, the $\mathrm{ONH}$ measurements are influenced by the blood flow of the ONH tissue (MT) $[15,16]$. To evaluate the major arteriovenous circulation 

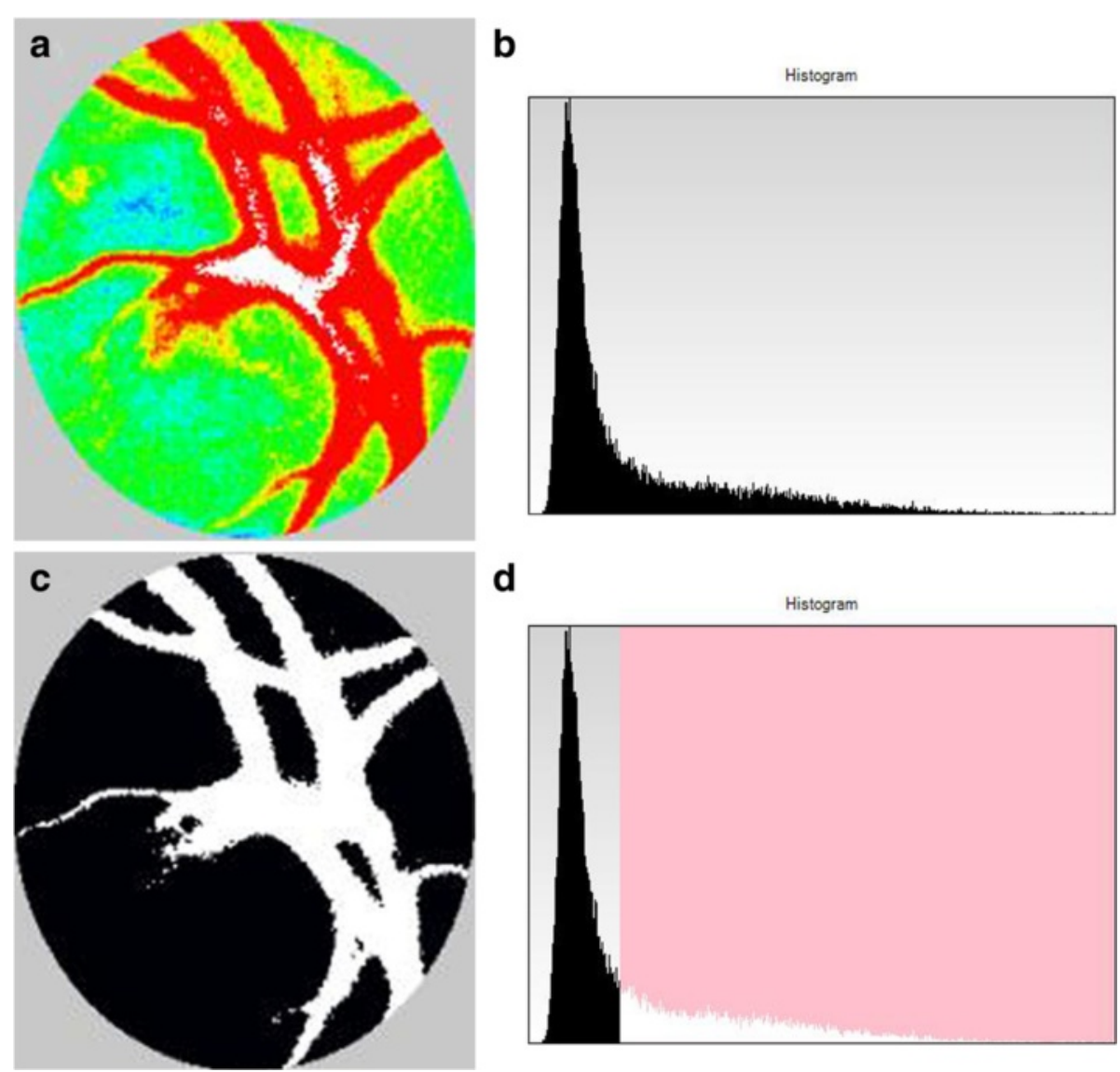

Fig. 3 Composite maps and histograms at the optic nerve head $(\mathrm{ONH})$ created by laser speckle flowgraphy. a: A false-color composite map at the $\mathrm{ONH}$. b: A histogramic analysis of the $\mathrm{ONH}$. The vertical axis represents the number of pixels and the horizontal axis represents the mean blur rate. c: A binary format image for segmentation between the vessel (white area) and tissue (black area) areas. $\mathbf{d}$ : A histogram analyzed using the built-in image viewer software that uses an automated definitive threshold. The area to the left of the threshold line corresponds to the ONH tissue (black area in $\mathbf{c}$ ), whereas the area to the right of the line corresponds to the $\mathrm{ONH}$ vessel (white area in c)

of the $\mathrm{ONH}$ excluding the blood flow of the $\mathrm{ONH}$ tissue, the value was obtained by subtracting the MT from the MV [11]. In addition, to exclude the influence of each patient's systemic circulation at the time of the measurements and to allow for inter-patient comparisons of the measurements, a corrected MBR value was obtained by the MBR (MV minus MT) of the affected eye divided by the MBR (MV minus MT) of the fellow eye [11]. The LSFG was measured at the baseline and at 1,3 , and 6 months after the initial treatment.

\section{Microperimetry}

The retinal sensitivity was determined by macular integrity assessment (MAIA) microperimetry (CenterVue, Padova, Italy; Figs. 1 and 2). MAIA testing was conducted in a dark room without pupil dilation. The testing conditions were similar to that described in detail in previous studies $[17,18]$. Briefly, 37 loci in the $10^{\circ}$ central macula was assessed using a 4 to 2 threshold strategy, a fixation target that consisted of a red circle with a $1^{\circ}$ diameter, stimulus size of Goldmann III, background luminance of 4 apostilb (asb), maximum luminance of $1000 \mathrm{asb}$, and a stimulus dynamic range of $36 \mathrm{~dB}$.

\section{Calculation of venous tortuosity index}

The venous tortuosity index was calculated as previously reported (Figs. 1 and 2) [20, 21]. Briefly, color fundus photographs were obtained with a Topcon fundus camera (TRC-50DX, Topcon, Tokyo, Japan). The course of the veins was traced using the Photoshop software (Adobe Systems, Inc. Ca, USA). Measurements of the superior and inferior venous arcades were obtained starting from the $\mathrm{ONH}$ margin to the crossing point of a circle whose diameter was the distance from the center of $\mathrm{ONH}$ to the fovea. NIH ImageJ software was used to measure the length of the tortuous vein and length of the chord of the vessel. The venous tortuosity was calculated by dividing the arc length of the retinal vein by the chord length of the same segment. The average of the venous tortuosity of the superior and inferior venous 
arcades was calculated to obtain the venous tortuosity index.

\section{Statistical analyses}

Repeated-measures analysis of variance (ANOVA) with Greenhouse-Geisser corrections was used to determine the significance of the changes in the BCVA, retinal sensitivity, corrected MBR, MV, MT, SBP, DBP, heart rate, IOP, MAP, and MOPP. The Bonferroni test was used for post hoc analysis. Pearson's correlation tests were used to determine the significance of correlations between the corrected MBR and the BCVA, retinal sensitivity, arteriovenous passage time, or venous tortuosity index. A $P$ value of $<0.05$ was considered statistically significant.

\section{Results}

All patients were followed for at least 6 months after the initial injection of IVB or IVR. IVB was performed on 15 eyes and IVR on 16 eyes. The injections were administered two to five times $(3.52 \pm 0.88$ (mean $\pm \mathrm{SD}))$ to each patient during the 6 months. According to the FA findings, the diagnosis of nonischemic CRVO was made in 26 eyes (Figs. 4 and 5), and the diagnosis of ischemic CRVO was made in 5 eyes. The SBP, DBP, heart rate, IOP, MAP, and MOPP were not significantly different among the baseline and 1,3 , and 6 months after the treatments $(P=0.930, P=0.958, P=0.966, P=0.745$, $P=0.969, P=0.886$, respectively).

At the baseline, the corrected MBR was $0.63 \pm 0.26$. The arteriovenous passage time on FA was $14.0 \pm 5.2 \mathrm{~s}$, and the venous tortuosity index on color fundus photograph was $1.113 \pm 0.053$. The corrected MBR was significantly correlated with the arteriovenous passage time $(r=-0.807, P<0.001 ;$ Fig. $6 a)$ and venous tortuosity

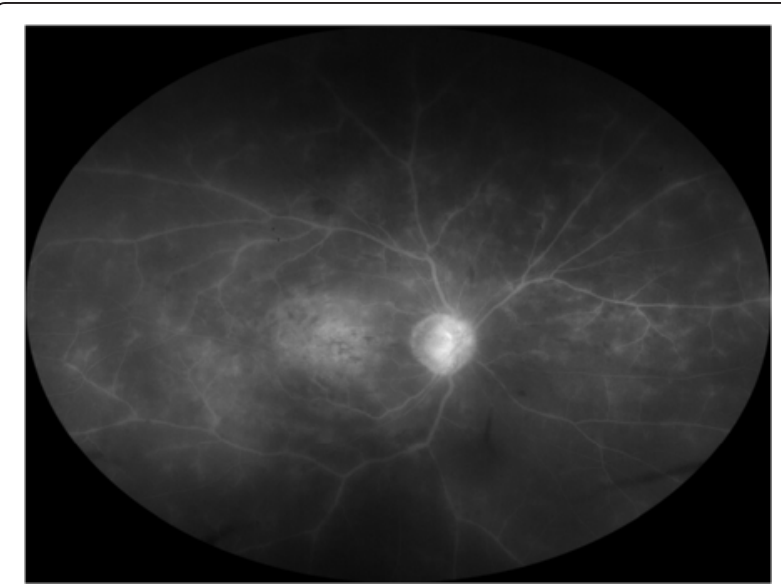

Fig. 4 Fluorescein angiography before anti-VEGF therapy for central retinal vein occlusion (CRVO). The same case presented in Fig. 1. Because there are not more than 10 disc areas of the nonperfusion areas, this case was diagnosed with nonischemic CRVO

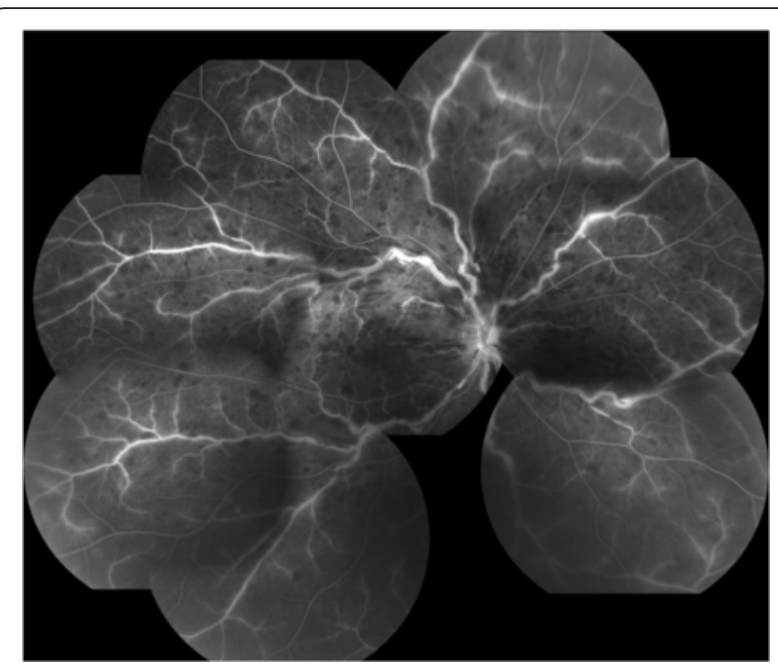

Fig. 5 Fluorescein angiography before anti-VEGF therapy for central retinal vein occlusion (CRVO). The same case presented in Fig. 2. Because there are not more than 10 disc areas of the nonperfusion areas, this case was also diagnosed with nonischemic CRVO

index $(r=-0.716, P<0.001)$. The corrected MBR after the first intravitreal injection was $0.60 \pm 0.23$ at 1 month, $0.61 \pm 0.23$ at 3 months, and $0.62 \pm 0.28$ at 6 months (Additional file 1). No significant differences were observed in the corrected MBR among the baseline and post-treatment times $(P=0.379)$. The $M V$ in the affected eye was $34.8 \pm 11.2$ at the baseline, $32.0 \pm 10.3$ at 1 month, $31.6 \pm 10.9$ at 3 months, and $32.9 \pm 12.2$ at 6 months. No significant differences were observed in the MV among the baseline and post-treatment times $(P=$ $0.116)$. The MT was $14.3 \pm 4.6$ at the baseline, $12.4 \pm 3.8$ at 1 month, $11.7 \pm 4.0$ at 3 months, and $12.3 \pm 4.2$ at 6 months. Significant differences were observed in the MT among the baseline and post-treatment times $(P=0.003)$. The MT at 1 and 3 months was significantly smaller than that at the baseline ( $P=0.011, P=0.002$, respectively).

The mean BCVA was 0.68 $\pm 0.48 \log$ MAR units at the baseline, $0.39 \pm 0.36 \log$ MAR units at 1 month after the first intravitreal injection, $0.36 \pm 0.35 \log$ MAR units at 3 months, and $0.33 \pm 0.42 \log M A R$ units at 6 months. Significant differences were observed in the BCVA among the baseline and post-treatment times $(P<0.001)$. The BCVA at 1,3 , and 6 months was significantly better than that at the baseline $(P<0.001, P=0.001, P=0.007$, respectively).

The retinal sensitivity was $15.2 \pm 7.5 \mathrm{~dB}$ at the baseline, $18.7 \pm 6.6 \mathrm{~dB}$ at 1 month, $20.9 \pm 6.0 \mathrm{~dB}$ at 3 months, and $20.4 \pm 6.0 \mathrm{~dB}$ at 6 months. Significant differences were observed in the retinal sensitivity among the baseline and post-treatment times $(P<0.001)$. The retinal sensitivity at 1,3 , and 6 months was significantly better than that at the baseline $(P=0.001, P<0.001, P=0.001$, respectively). 


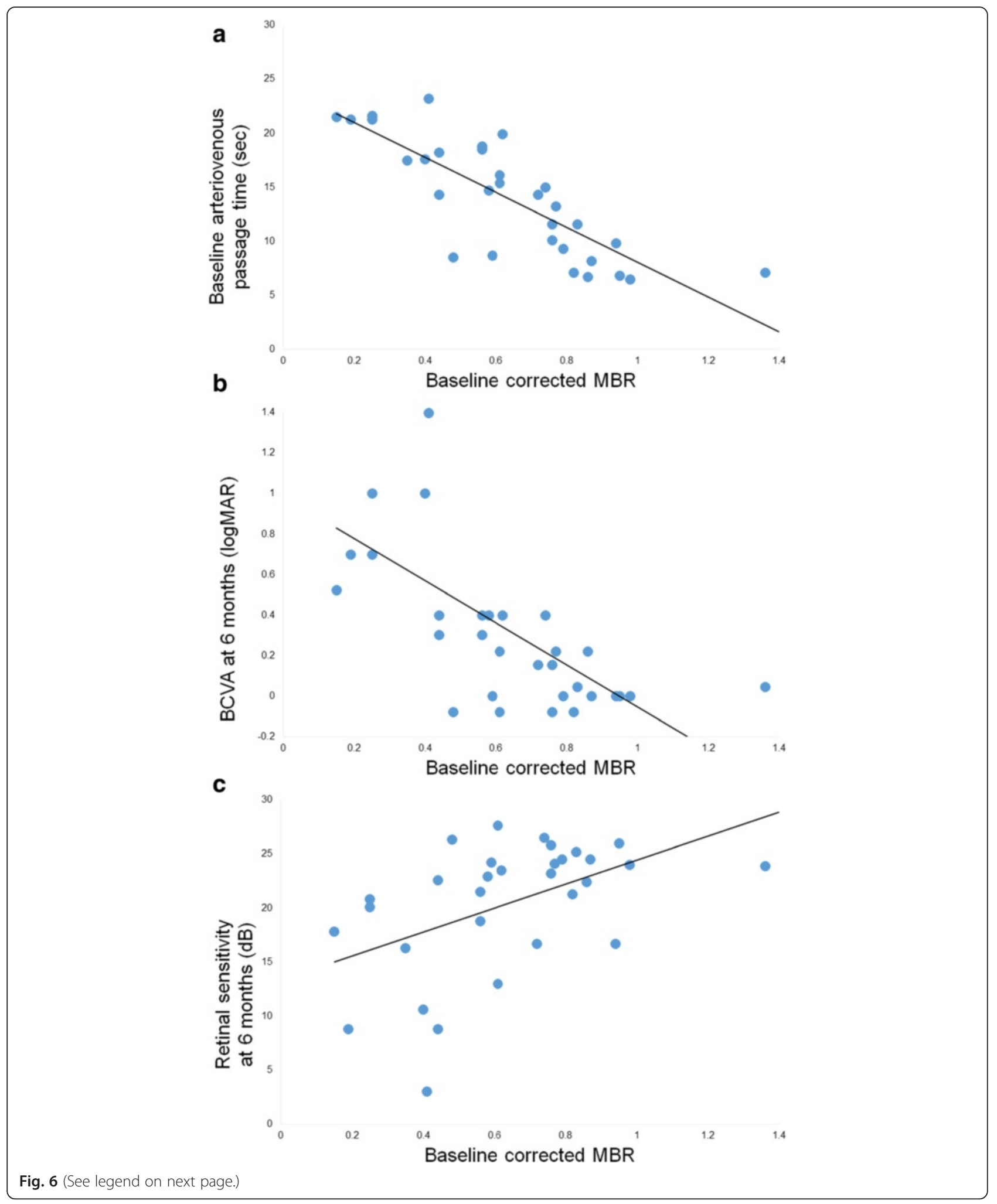


(See figure on previous page.)

Fig. 6 Correlation between optic nerve head $(\mathrm{ONH})$ circulation and arteriovenous passage time or visual function. Correlation between the baseline mean blur rate (MBR) at the $\mathrm{ONH}$ and the baseline arteriovenous passage time, the best-corrected visual acuity (BCVA) at 6 months, or mean retinal sensitivity at 6 months is presented. To exclude the influence of systemic circulation and blood flow of ONH tissue, the corrected MBR was calculated as; (MBR of ONH vessel area - MBR of ONH tissue area) in the affected eye $\div$ (vascular MBR - tissue MBR) in the healthy fellow eye [11]. a: Correlation between the baseline corrected MBR at the $\mathrm{ONH}$ and the arteriovenous passage time at the baseline. The corrected MBR is significantly correlated with the arteriovenous passage time on fluorescein angiography $(r=-0.807, P<0.001)$. The solid line represents the linear regression line $(y=-16.102 x+24.200)$. $\mathbf{b}$ : Correlation between the baseline corrected MBR at the ONH and BCVA in logMAR units at 6 months after the initial treatment. The corrected MBR is significantly correlated with the BCVA at 6 months $(r=-0.651, P<0.001)$. The solid line represents the linear regression line $(y=-1.036 x+0.983)$. c: Correlation between the baseline corrected MBR at the ONH and mean retinal sensitivity at 6 months. The corrected MBR is significantly correlated with the retinal sensitivity at 6 months $(r=0.485, P=0.005)$. The solid line represents the linear regression line $(y=11.059 x+13.378)$

The MBR was significantly correlated with the BCVA (all $P<0.005$ ) and the retinal sensitivity (all $P<0.050$, Table 1) at all time points.

There was a significant correlation between the corrected MBR at the baseline and the BCVA at 6 months $(r=-0.651, P<0.001$; Fig. $6 \mathrm{~b})$. There was also a significant correlation between the corrected MBR at the baseline and the retinal sensitivity at 6 months $(r=0.485$, $P=0.005$; Fig. 6c).

\section{Discussion}

The results showed that the corrected MBR at the baseline was significantly correlated with the BCVA and retinal sensitivity at 6 months after anti-VEGF therapy. This indicates that the visual prognosis is good in patients with CRVO who have good blood flow on the $\mathrm{ONH}$ at the baseline. In a report on 3 cases with CRVO, Matsumoto et al. also suggested that the prognosis of CRVO may be predicted by measuring the MBR using LSFG [13]. In eyes with ME due to a BRVO, Nagaoka et al. reported that the visual prognosis was good when the retinal blood flow measured by laser Doppler velocimetry before IVB was good [12]. Taken together, these results suggest that the status of the blood flow prior to the treatment is a useful predictor of the post-treatment BCVA for both CRVO and BRVO.

Table 1 Correlations between MBR on $\mathrm{ONH}$ and BCVA or retinal sensitivity before and after anti-VEGF therapy

\begin{tabular}{lllll}
\hline & \multicolumn{5}{l}{ Corrected MBR on ONH } \\
\cline { 2 - 5 } & Baseline & 1 month & 3 months & 6 months \\
\hline BCVA (logMAR) & $r=-0.543$ & $r=-0.672$ & $r=-0.546$ & $r=-0.565$ \\
& $P=0.001$ & $P<0.001$ & $P=0.001$ & $P<0.001$ \\
Retinal sensitivity (dB) & $r=0.572$ & $r=0.608$ & $r=0.552$ & $r=0.430$ \\
& $P<0.001$ & $P<0.001$ & $P=0.001$ & $P=0.015$
\end{tabular}

BCVA, best corrected visual acuity; Corrected MBR, (MBR of ONH vessel area - MBR of ONH tissue area) in the affected eye $\div$ (vascular MBR - tissue MBR) in the unaffected eye; logMAR, logarithm of the minimum angle of resolution; MBR, mean blur rate; $\mathrm{ONH}$, optic nerve head; $\mathrm{VEGF}$, vascular endothelial growth factor
The measurements of the LSFG had excellent reproducibility which then permitted a non-invasive method to measure the ocular circulation. Aizawa et al. used LSFG and reported that the intra-session reproducibility of the MBR in the $\mathrm{ONH}$ of three continuous examinations was excellent with a coefficient of variation of $3.4 \pm 2.0$ and an intraclass correlation coefficient of 0.95 [9]. Aizawa et al. also reported that the MBR of the $\mathrm{ONH}$ tissue was strongly correlated with the visual field sensitivity in eyes with glaucoma [3]. In addition, Maekubo et al. reported that measurements of the $\mathrm{ONH}$ circulation by LSFG could be used for differentiating nonarteritic ischemic optic neuropathy from anterior optic neuritis [19]. Based on these findings, measuring the ONH circulation by LSFG can be considered a useful method in the clinic.

Yamada et al. measured the MBR on the $\mathrm{ONH}$ in eyes with untreated CRVO [11]. The corrected MBR was calculated to exclude the influence of the systemic circulation and blood flow of the $\mathrm{ONH}$ tissue. After obtaining the MBR values by subtracting the MT from the MV, the $\mathrm{ONH}$ blood flow was evaluated by taking the MBR values of the affected eye divided by the MBR values of the healthy fellow eye. The authors reported that this corrected MBR was significantly correlated with the arteriovenous passage time [11]. Similarly, a significant correlation was found between the corrected MBR and arteriovenous passage time in the present study. The strong correlation between the corrected MBR and arteriovenous passage time, which has been used as an indicator of retinal circulation, confirms that the method we used is a highly reliable way to evaluate the circulatory status of the ONH. In addition, Yamada et al. reported that the corrected MBR values in large $\mathrm{ONH}$ vessels were significantly correlated with the aqueous VEGF concentrations in eyes with CRVO [11].

It is sometimes difficult to distinguish the vessel and tissue areas at the $\mathrm{ONH}$ in eyes with CRVO, because dilatation or blood stasis of capillary vessel on $\mathrm{ONH}$ obscures the boundary between the large vessel and tissue 
on the LSFG images. Thus, the MV and MT values measured in eyes with CRVO may include a measurement error. However, the corrected MBR in the present study was significantly correlated with not only arteriovenous passage time on FA but also the venous tortuosity index on fundus photograph, which suggests that this measurement error is considered to be small.

Nitta et al. reported that there was no significant change in the MBR measured by LSFG in the retinal artery, retinal vein, or $\mathrm{ONH}$ after a single IVB injection in eyes with a BRVO [22]. Nagaoka et al. also reported no significant changes in the retinal blood flow measured by laser Doppler velocimetry after a single IVB injection for BRVO [12]. Consistent with these findings, our results showed no significant differences in the corrected MBR after IVB or IVR in eyes with a CRVO. On the other hand, Matsumoto et al. reported 3 cases of ME secondary to nonischemic CRVO in which the blood flow increased after IVBs [13]. In their case, the MBR increased in 3 or 4 weeks after the IVB was confirmed by LSFG, but long term changes of the MBR and exact relationship between the MBR and visual prognosis were unclear.

This study has several limitations. First, the sample size was small, and the follow-up period was short. Further studies with a larger sample size and longer follow-up periods would be required to confirm our findings. We also need to keep in mind that when using LSFG the MBR is a relative value of the blood flow velocity and not the absolute value. Therefore, when comparing the difference between eyes, we used the corrected MBR value. The other limitation is that the present study included both patients treated with IVB and those with IVR. Lastly, Mayama et al. reported that phenylephrine eye drops can depress the ONH circulation [23]. In our study, Mydrin P, tropicamide and phenylephrine, was used prior to the LSFG testing to obtain more accurate measurements of the $\mathrm{ONH}$ circulation with LSFG. Thus, there is a possibility that phenylephrine may have influenced the $\mathrm{ONH}$ circulation. However, because Mydrin P was administered to both the affected and unaffected eyes and ONH circulation was evaluated using the equation; the MBR of the affected eye/MBR of the unaffected eye, the magnitude of this influence may be small.

\section{Conclusions}

The blood flow velocity on the $\mathrm{ONH}$ was significantly correlated with the BCVA and retinal sensitivity before and after anti-VEGF therapy for CRVO. We suggest that the blood flow velocity of the ONH before the therapy is a predictive factor for the visual outcome after treatment for CRVO.

\section{Ethics and consent to participate}

Approval was obtained from the Institutional Review Board of Saneikai Tsukazaki Hospital prior to beginning this study, and the patients gave their written informed consent prior to their inclusion. The procedures used in this study adhered to the tenets of the Declaration of Helsinki.

\section{Consent to publish}

The patients have provided permission to publish clinical data of their case in this study.

\section{Additional file}

Additional file 1: Raw data (MBR, visual function). (DOC 68 kb)

\section{Abbreviations}

asb: apostilb; BCVA: best-corrected visual acuity; BRVO: branch retinal vein occlusion; CRVO: central retinal vein occlusion; DBP: diastolic blood pressure; FA: fluorescein angiography; IOP: intraocular pressure; IVB: intravitreal bevacizumab; IVR: intravitreal ranibizumab; LSFG: laser speckle flowgraphy; MAIA: macular integrity assessment; MAP: mean arterial pressure; MBR: mean blur rate; ME: macular edema; MOPP: mean ocular perfusion pressure; MT: mean blur rate of tissue area; MV: mean blur rate of vessel area; OCT: optical coherence tomography; ONH: optic nerve head; SBP: systolic blood pressure; SD-OCT: spectral-domain optical coherence tomography; UMIN: University hospital Medical Information Network; VEGF: vascular endothelial growth factor.

\section{Competing interests}

The authors declare that they have no competing interests.

\section{Authors' contributions}

Design of the study (YM), conduct of the study (DN, TN, YY, HT), management of the data (DN, YM, KS, KA, TN, YY, HT), analysis of the data (DN, YM, KS, KA, YK), interpretation of the data (DN, YM, KS, KA, YK), preparation of the manuscript (DN, YM) and overall coordination (YK). All authors read and approved the final manuscript.

\section{Acknowledgements}

This work was supported in part by Grants-in-Aid 25462717 (to YM) from the Ministry of Education, Culture, Sports, Science and Technology, Japan. The funding agencies had no role in the study design, data collection and analysis, decision to publish, or preparation of the manuscript. The authors thank Professor Emeritus Duco Hamasaki of the Bascom Palmer Eye Institute of the University of Miami for providing critical discussions and suggestions for our study and revision of the final manuscript.

\section{Author details}

'Department of Ophthalmology, Saneikai Tsukazaki Hospital, Himeji, Japan. ${ }^{2}$ Department of Ophthalmology and Visual Sciences, Graduate School of Biomedical Sciences, Hiroshima University, Hiroshima, Japan. ${ }^{3}$ Department of Ophthalmology, Institute of Biomedical Sciences, Tokushima University Graduate School, 3-18-15 Kuramoto, Tokushima 770-8503, Japan.

Received: 21 October 2015 Accepted: 29 March 2016

Published online: 05 April 2016

References

1. Yeh S, Kim SJ, Ho AC, Schoenberger SD, Bakri SJ, Ehlers JP, et al. Therapies for macular edema associated with central retinal vein occlusion: a report by the American Academy of Ophthalmology. Ophthalmology. 2015;122(4):769-78.

2. Sugiyama T, Araie M, Riva CE, Schmetterer L, Orgul S. Use of laser speckle flowgraphy in ocular blood flow research. Acta Ophthalmol. 2010;88(7):723-9.

3. Aizawa N, Kunikata H, Yokoyama Y, Nakazawa T. Correlation between optic disc microcirculation in glaucoma measured with laser speckle flowgraphy 
and fluorescein angiography, and the correlation with mean deviation. Clin Exp Ophthalmol. 2014;42(3):293-4.

4. Iwase T, Ra E, Yamamoto K, Kaneko H, Ito Y, Terasaki H. Differences of retinal blood flow between arteries and veins determined by laser speckle flowgraphy in healthy subjects. Medicine (Baltimore). 2015;94(33):e1256.

5. Yanagida K, Iwase T, Yamamoto K, Ra E, Kaneko H, Murotani K, et al. Sex-related differences in ocular blood flow of healthy subjects using laser speckle flowgraphy. Invest Ophthalmol Vis Sci. 2015;56(8):4880-90.

6. Iwase T, Yamamoto K, Ra E, Murotani K, Matsui S, Terasaki H. Diurnal variations in blood flow at optic nerve head and choroid in healthy eyes: diurnal variations in blood flow. Medicine (Baltimore). 2015;94(6):e519.

7. Takahashi H, Sugiyama T, Tokushige H, Maeno T, Nakazawa T, Ikeda T, et al. Comparison of CCD-equipped laser speckle flowgraphy with hydrogen gas clearance method in the measurement of optic nerve head microcirculation in rabbits. Exp Eye Res. 2013;108:10-5.

8. Wang L, Cull GA, Piper C, Burgoyne CF, Fortune B. Anterior and posterior optic nerve head blood flow in nonhuman primate experimental glaucoma model measured by laser speckle imaging technique and microsphere method. Invest Ophthalmol Vis Sci. 2012;53(13):8303-9.

9. Aizawa N, Yokoyama Y, Chiba N, Omodaka K, Yasuda M, Otomo T, et al. Reproducibility of retinal circulation measurements obtained using laser speckle flowgraphy-NAVI in patients with glaucoma. Clin Ophthalmol. 2011;5:1171-6

10. Sugiyama T, Kojima S, Ishida O, Ikeda T. Changes in optic nerve head blood flow induced by the combined therapy of latanoprost and beta blockers. Acta Ophthalmol. 2009:87(7):797-800.

11. Yamada Y, Suzuma K, Matsumoto M, Tsuiki E, Fujikawa A, Harada T, et al. Retinal blood flow correlates to aqueous vascular endothelial growth factor in central retinal vein occlusion. Retina. 2015;35(10):2037-42.

12. Nagaoka T, Sogawa K, Yoshida A. Changes in retinal blood flow in patients with macular edema secondary to branch retinal vein occlusion before and after intravitreal injection of bevacizumab. Retina. 2014;34(10):2037-43.

13. Matsumoto M, Suzuma K, Fukazawa Y, Yamada Y, Tsuiki E, Fujikawa A, et al. Retinal blood flow levels measured by laser speckle flowgraphy in patients who received intravitreal bevacizumab injection for macular edema secondary to central retinal vein occlusion. Retin Cases Brief Rep. 2014;8(1):60-6.

14. Group CVOS. Natural history and clinical management of central retinal vein occlusion. The Central Vein Occlusion Study Group. Arch Ophthalmol. 1997;115(4):486-91.

15. Isono H, Kishi S, Kimura Y, Hagiwara N, Konishi N, Fujii H. Observation of choroidal circulation using index of erythrocytic velocity. Arch Ophthalmol. 2003;121(2):225-31.

16. Nagahara M, Tamaki Y, Tomidokoro A, Araie M. In vivo measurement of blood velocity in human major retinal vessels using the laser speckle method. Invest Ophthalmol Vis Sci. 2011;52(1):87-92.

17. Sato S, Hirooka K, Baba T, Tenkumo K, Nitta E, Shiraga F. Correlation between the ganglion cell-inner plexiform layer thickness measured with cirrus HD-OCT and macular visual field sensitivity measured with microperimetry. Invest Ophthalmol Vis Sci. 2013;54(4):3046-51.

18. Roisman L, Ribeiro JC, Fechine FV, Lavinsky D, Moraes N, Campos M, et al. Does microperimetry have a prognostic value in central serous chorioretinopathy? Retina. 2014;34(4):713-8.

19. Maekubo T, Chuman H, Nao-I N. Laser speckle flowgraphy for differentiating between nonarteritic ischemic optic neuropathy and anterior optic neuritis. Jpn J Ophthalmol. 2013;57(4):385-90.

20. Ferrara DC, Koizumi H, Spaide RF. Early bevacizumab treatment of central retinal vein occlusion. Am J Ophthalmol. 2007;144(6):864-71.

21. Yasuda S, Kachi S, Kondo M, Ueno S, Kaneko H, Terasaki H. Significant correlation between retinal venous tortuosity and aqueous vascular endothelial growth factor concentration in eyes with central retinal vein occlusion. PLoS One. 2015:10(7):e0134267.

22. Nitta F, Kunikata H, Aizawa N, Omodaka K, Shiga Y, Yasuda M, et al. The effect of intravitreal bevacizumab on ocular blood flow in diabetic retinopathy and branch retinal vein occlusion as measured by laser speckle flowgraphy. Clin Ophthalmol. 2014;8:1119-27.

23. Mayama C, Ishii K, Saeki T, Ota T, Tomidokoro A, Araie M. Effects of topica phenylephrine and tafluprost on optic nerve head circulation in monkeys with unilateral experimental glaucoma. Invest Ophthalmol Vis Sci. 2010;51(8):4117-24.

\section{Submit your next manuscript to BioMed Central and we will help you at every step:}

- We accept pre-submission inquiries

- Our selector tool helps you to find the most relevant journal

- We provide round the clock customer support

- Convenient online submission

- Thorough peer review

- Inclusion in PubMed and all major indexing services

- Maximum visibility for your research

Submit your manuscript at www.biomedcentral.com/submit
Biomed Central 